\title{
The Exoskeleton Robot Research Progress and Prospect
}

\author{
Bing wang \\ Mechanical engineering school \\ huaihai institute of technology \\ Lianyungang, China \\ e-mail: hrbwb2001@163.com
}

\author{
Yanjun zhao \\ Mechanical engineering school \\ Shandong university of science and technology \\ zibo, China \\ e-mail: zyj627010@163.com
}

\begin{abstract}
The exoskeleton robot is essentially a wearable robot, it will be the person's intelligence and the external mechanical equipment of mechanical energy together, which can give a person the power and provide additional capabilities, enhance the human body function. This article summarizes the origin of the exoskeleton robot technology, the research progress, and combined with the current research direction in the future is prospected, exoskeleton robot plays an irreplaceable function in the society to use. Exoskeleton robot in such aspects is as wear resistance, intelligent and portability are the development of the breakthrough, but should strengthen the micro energy, lightweight portable materials, multi-functional system, such as research and development, can be widely used in military, scientific research, tourism, transportation, disaster relief and other fields. Exoskeleton is a combination of human intelligence and mechanical power device of the mechanical energy of a combination of human and robot, it can help people bearing by load, effectively strengthen the load ability, help people walk in a variety of complex terrain.
\end{abstract}

\begin{tabular}{llr}
\multicolumn{1}{c}{ Keywords- } & \multicolumn{1}{c}{$\begin{array}{l}\text { Exoskeleton } \\
\text { mechanical;Energy } \\
\text { combination;load ability }\end{array}$}
\end{tabular} The $\begin{array}{r}\text { robot;Intelligent } \\
\text { man-machine }\end{array}$

\section{INTRODUCTION}

Modern robots have mechanical power device which makes the robot can easily complete many difficult tasks, such as lifting, handling the heavy load, etc. Although modern robot control technology has come a long way development, it is still far short of person's intelligence level, including the decision-making ability And the perception of the environment. At the same time, the human beings of wisdom is unmatched by any biological and mechanical device. Therefore, people will be smart and have strong robot mechanical energy, integrated into a system that will lead to large change, which is the exoskeleton robot design thought.[1]

The exoskeleton robot is essentially a wearable robot [2], the term Exoskeleton comes from the biology exoskeleton. In the interpretation of the biology and bone for: provide biological protection and support the hard outer structure, such as crustaceans or kun worm shell. Exoskeleton is an important component of invertebrates points, is of great significance for invertebrates, it can support the body, to protect the vital organs of the body, to provide agile movement skills, and to feel the environment, many important sense organs are attached on the exoskeleton. The exoskeleton has multiple advantages which caused the great interest of scientists and began to study artificial exoskeleton from the perspective of bionics.

The exoskeleton robot is not only a kind of artificial exoskeleton but also a kind of mechanical mechanism wearing in the operator which provide the operator with the external body, such as protection, physical support such as function, at the same time, combined with sensing, control, drive, information such as robotics, enables the exoskeleton to complete certain functions and tasks under the operator's control.

\section{THE TYPE AND STRUCTURE OF THE EXOSKELETON ROBOT}

Exoskeleton robot is a combination of human intelligence and mechanical power plant combining human with mechanical energy wearable equipment. Structurally, the exoskeleton robot can be roughly divided into upper limb exoskeleton robot, lower limb exoskeleton robot, general exoskeleton robot and all kinds of joint correction or restorative training outside the joints bone robot. From the function division, exoskeleton device can be roughly divided into two types: the first is to assist and rehabilitation of exoskeleton robot, for example assist the disabled or elderly people, the exoskeleton of walking robot, auxiliary body damage or motor function in patients with partial loss of rehabilitation treatment and rehabilitation training practiced the exoskeleton robot. Another is to enhance normal power, speed, negative type of power such as the function of the body weight and endurance exoskeleton robot. In recent years, many developed countries have lauched the exoskeleton manufacture, and gradually applied to military operations auxiliary equipment, medical equipment, power equipment, and other fields, including the United States and Japan bone robot research achievements on the most significant. 


\section{A. Upper limb exoskeleton robot}

The main functions of the human upper extremity is, fetching and elevated operation, so the design limb exoskeleton robot to consider shoulder, elbow, wrist and upper arm, forearm and hand such as kinematics element and the degrees of freedom of flexible operation of human upper limb and so on many kinds of characteristics.

The earliest study of exoskeleton system began in the 1960s[3]. In 1962, the United States air force requires Cornell air lab for one using master-slave control mode of the feasibility research of human amplifier system investigation[4]. From 1960 to 1971, American general electric company start to study a exoskeletons prototype based on master-slave control, named "Hardiman" which is shown in Figure 1. .

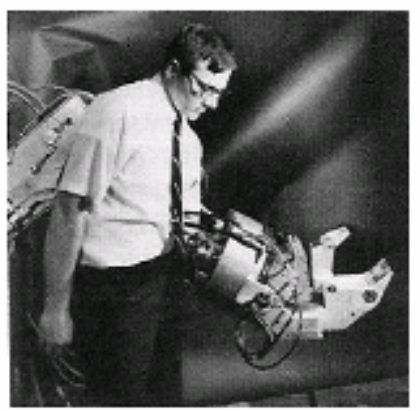

Figure 1. Hardiman exoskeleton system

Hardiman using motor drive, can like lift 10 pounds to lift a weight of 250 pounds. However, due to technical limit system, Hardiman implements only one arm control, grind to a halt the development. At the same time exoskeleton study and Belgrade Vukobratovic et al, of the university of their research is mainly used for auxiliary help paralyzed patients exercise rehabilitation of lower limbs[5-6].

Although only points movement forms, research in the process of balancing algorithm in pairs. The foot of walking robot has been widely applied. In the $1990 \mathrm{~s}$, because of sensing technology, material technology and the development of control technology, foreign study of bone, large-scale exhibition open it. The exoskeletons of early only as a man-machine interface to use, As the master manipulator robot teleoperation system[7], the human body arm or finger position detection, etc[8-9]

In the mid 90s, and force feedback technology tactile feedback technology is widely used and exoskeleton system. Using force after feedback technology, is more authenticity of teleoperation, control effect fruit is greatly improved than before, exoskeleton in many fields application [8]. After entering the 21st century, because of energy technology, micro driver technology, materials science, the development of information technology, various types of outside bones have emerged, the exoskeleton also gradually to the practical direction development, such as Japan Cybernics laboratory developed the tsukuba university The world's first commercial exoskeleton robot Hybrid Assistive Legs (HAL). This device can help the disabled to $4 \mathrm{~km} / \mathrm{h}$ speed walking, effortlessly climb the stairs[10 to 12$]$

\section{B. Lower limb exoskeleton robot}

Human lower limb main bear standing, balancing, walking, and other functions, its movement structure is simpler than upper limbs. Design of lower limb exoskeleton need larger joints lose out, want to consider gait stability, balance, and its mechanical structure design and control algorithm and so on is more complicated. In 2000, America's defence advanced research projects agency (defense advanced research projects agency, DARPA) at the university of California, Berkeley human body engineering laboratory (HEL), SARCOS, oak ridge national real robot company Laboratory (ORNL) and the Millennium Jet company conducted power exoskeletons machine, etc

In 2004, the United States at the university of California, Berkeley, developed the exoskeleton of the lower limbs Robot (Berkeley lower extremity exoskeleton, BLEEX) (as shown in figure 4), it is America's defence advanced research projects agency (DARPA) EHPA project developed the first affordable and bringing the exoskeleton robot mobile power supply. BLEEX exoskeleton robot consists of power equipment, backpacks timbering, 2 biomimetic mechanical power legs, adopts hydraulic drive, the hydraulic pump energy comes from the backpack timbering in hydraulic transmission system and the box miniature space velocity sensor which is shown in Figure 2. A complete set of equipment quality is about $45 \mathrm{~kg}$, subjects on the equipment, to carry a quality up to $35 \mathrm{~kg}$ Backpack, still can walk freely.

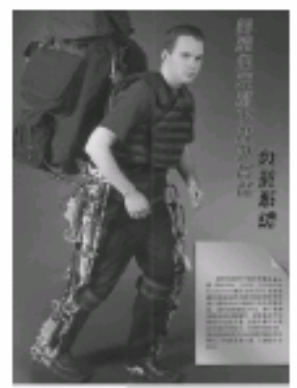

Figure 2. BLEEX exoskeleton robot

\section{The whole body exoskeleton robot}

In 2008,Steve Jacobsen, companies in the United States and others developed successfully XOS-1 exoskeleton robot, as shown in figure 6. The device type for the whole body exoskeleton robot, using the body of the sensor can quickly effectively respond to the behavior of the wearer action. When the users put on XOS - 1, 200 lb (1 lb $6=0.453 \mathrm{~kg}$ ) of weight is easily for 50-500 times in a row. The equipment bring their own electricity as the source of power, but can only be used for $40 \mathrm{~min}$.

In 2010 Thor again issued the second generation of exoskeleton mechanical XOS - 2, the device also is the whole body exoskeleton robot, which is the first generation improved version, lighter, faster and stronger than the first generation, at the same time power consumption will be reduced $50 \%$. XOS 2 consists of a series of structure, sensor, actuator and controller driven by high pressure hydraulic. With the aid of the 
exoskeleton, the wearer will lifte easily $200 \mathrm{lb}$ weight hundreds of times and won't feel tired, still can repeat puncture 3 in $(1=2.54 \mathrm{~cm})$ in thick planks. The device is very light, the soldiers in it can be finished playing football, hit speed ball, climb stairs and downhill[10].

\section{KEY TECHNIQUES IN THE EXOSKELETON ROBOT}

\section{A. exoskeletons mechanical structures}

Exoskeleton robot is a kind of mechanism of human wearable, which safety, comfort and practicality should be first consider factors. Exoskeleton robot can be roughly divided into the upper limb, lower limb exoskeleton robot machine apparatus and the whole body exoskeleton robot, from which an exoskeleton robot will be "Close contact" with the human body, so the exoskeletons to all mechanical structure design surface analysis of human body each joint range of motion and movement characteristics, reasonable as far as possible, anthropomorphic, and safe and comfortable, flexible motion, and the wearer is not restricted.

\section{B. the exoskeleton robot drive system}

Exoskeleton robot drive system and the drive must be lightweight, small size, and can provide enough driving moment and torque, at the same time to have good dispersion thermal performance. The exoskeleton of the commonly used on current international drive system mainly has electricity motor drive system, three kinds of pneumatic drive system, hydraulic drive system. Motor drive system, three kinds of pneumatic drive system, hydraulic drive system.

1) hydraulic drive

Hydraulic drive is a fluid as working medium for energy transfer and control transmission ways. Hydraulic system with inertia small, simple structure, high reliability and work. The advantages of stable. But work pressure liquid easy to leak, and noise large, low energy efficiency, low transmission speed. The university of California, Berkeley The school developed into power machinery clothing and Raytheon BLEEX series and outside The bone mechanical loading XOS series adopts hydraulic drive way to drive the exoskeleton.

\section{2) pneumatic driving}

Pneumatic drive is compressed air as working medium for energy transfer and control

the mode of transmission. Pneumatic system has a simple structure, no pollution, resistance loss small, low cost advantages, but the stability of pneumatic transmission speed is poorer, letter transmission speed is slow, poor control, is not suitable for high power transmission system. The kanagawa type body exoskeleton robot developed successful engineering university (power Assist suit, PAS) USES pneumatic transmission device can will increase the power of the consumer0.5 1. 0 times.

3) motor drive

Motor drive system use electric power equipment and adjust the electrical parameter a way of transmission of power and control. Motor system has mature technology, simple structure, no pollution, signal transmission quick and easy to realize automation, but the move poor state of balance, great quality, big inertia, the reversing is slow. Outside the Japanese atthe university of standing wave bone skeletons robot HAL series adopt the motor drive technology.

4) energy

At present, the exoskeleton robot mainly by the battery power supply, mobile range is Battery capacity and efficiency of the limit, how to improve the battery capacity of unit volume And the use efficiency of exoskeleton is scientific research institutions at home and abroad have been trying to solve.

\section{Controlling Methods}

Exoskeleton robot is the biggest difference is that it and other robots. The operator is a person, not by machine, the operator in the loop," People (Man in Loop) in the Loop", the operator and the exoskeleton has the real physical contact, formed a man-machine coupling the integration of system. The man-machine coupling system control objective is to make people and the machine can work harmoniously, to complete the task.

\section{A. The operator control}

Exoskeleton has a lot of is based on the purpose of rehabilitation has the external energy Gait rectification device driver. These devices are mainly lower limb exoskeleton, Used to support the weight of lower limb rehabilitation training for operators. This some device command signal usually comes from a healthy body. For example, By Yano design used in the exoskeleton of a switch and the ground reaction force sensor, through the operator control, drive the hip joint

In sports, change the distance of the foot off the ground. In view of the collapsed Risk, amputation, and patients with lower limb paralysis, auxiliary device designed a kind of movement, by finger joints sensor to control the movement of each leg. Hand refers to the movement of the monitoring by $6 \sim 8$ goniometer, found the fingers off section of the movement and the corresponding leg joints a one-to-one mapping. Its controlling characteristics by installing a set of switch in the hand. Use the motion of the forearm to control the motion of the legs, also now the function of bearing. The downside of this approach is that the operator upper body can only used to command, not for other activities, and the operator must be continuously kept command, not only wasted strength, and the operator has become very unnatural.

\section{DELEOPMENT PROSPECTS}

Exoskeleton robot has a good development prospect in many fields. In recent years, the countries value exoskeleton robot technology breakthrough show, but still there are many key problems to be solved: (1) the larger volume, lumbering, its poor environmental adaptability and the flexibility of movement; (2) and the operator of the expected move don't match; (3) the exoskeleton robot's energy efficiency is not high; (4) control system of 
adaptive and self-learning ability is weak. In addition, the exoskeleton robot

To really into practice, consider the quality problems of its own, flexible security asked problem, noise problem, comfort, etc. With the progress of science and technology, all kinds of special function of exoskeleton robot will be widely applied in civil rehabilitation treatment and military power equipment.

\section{ACKNOWLEDGMENT}

This work is a project supported by Natural Science Foundation of Shandong under the grant No. ZR2011EL013.

\section{REFERENCES}

[1] Aaron M Dollar, Hugh Herr. Lower Extremity Exoskeletons and Active Orthoses: Challenges and Stateof-the-Art. IEEE Transactions on Robotics, 2008, 24 (1) : 144-158.

[2] Yang C J, Zhang J F, Chen Y, et al. A review of exoskeleton-type systems and their key technologies. Mechanical Engineering Science, 2008, 222(8):1599-1612. [3] Kazerooni H. Human augmentation and exoskeleton systems in berkeley. International Journal of Humanoid Robotics, 2007, 4(3) : 575-605.

[4] Kazerooni H, Steger R, Li H H. Hybrid Control of the Berkeley Lower Extremity Exoskeleton (BLEEX). The International Journal of Robotics Research, 2006, 25 (5) : 561-573.

[5] Chu A. Design of the Berkeley Lower Extremity Exoskeleton ( BLE - EX ) . Berkeley : University of Californnia, 2005: 16-32.
[6] Kazuo, Katubedda, Kiguchi K, et al. Mechanical Designs of Active Upper-Limb Exoskeleton Robots Stateof-the-Art and Design Difficulties. Proceedings of IEEE 11th International Conference on Rehabilitation Robotics. Japan: Kyoto International Conference Center, 2009: 178-187.

[7] Liu X P, Low K H. Development and Preliminary Study of the NTU Lower Extremity Exoskeleton. Proceedings of IEEE Conference on Cybernetics and Intelligent Systems. Singapore : Cybernetics and Intelligent Systems， 2004， 2: 1 243-1 247.

[8] Hayden, Kevin Cudby. Rex The Robotic Exoskeleton [EB/OL]. ( 2012-02-09 ) [2012-03-02]. http : //technology.tki.org.nz/Resour-ces/Casestudies/Technologists-practice-case-studies/Electronicsand-control-technologies/Rex-the-robotic-exoskeleton.

[9] Sakurai T, Sankai Y, et al. Development of motion instruction system with interactive robot suit HAL. Proceedings of IEEE International Conference on Robotics and Biomimetics, Japan : Robotics and Biomimetics (ROBIO) , 2009: 1 141-1 147.

[10] Yoshiyuki Sankai.Wearing Type Behavior Help Device Calibration Device and Control Progarm: USA, 2011000432Al. 2011-01-06.

[11] Yoshiyuki Sankai. Centroid Position Detector Device and Wearing Type Action Assistance Device Including Centroid Position Detector Device : USA , 20100271051A1. 2010-10-28.

[12] Yamamoto K, Ishii M, Noborisaka H, et al. Stand alone wearable power assisting suit-sensing and control systems//Proceedings of 13th IEEE International Workshop. Roman : Robot Human Interactive Communication, 2004: 661-666. 